University of Wollongong

Research Online

Faculty of Engineering and Information

Faculty of Engineering and Information

Sciences - Papers: Part A

Sciences

$1-1-2014$

A sensitivity analysis toolkit for the simplification of MV distribution network voltage management

\author{
Fabian Tamp \\ University of Wollongong, ft064@uowmail.edu.au \\ Philip Ciufo \\ University of Wollongong, ciufo@uow.edu.au
}

Follow this and additional works at: https://ro.uow.edu.au/eispapers

Part of the Engineering Commons, and the Science and Technology Studies Commons

Research Online is the open access institutional repository for the University of Wollongong. For further information contact the UOW Library: research-pubs@uow.edu.au 


\title{
A sensitivity analysis toolkit for the simplification of MV distribution network voltage management
}

\author{
Abstract \\ As distribution networks become increasingly variable and generation becomes increasingly \\ decentralised, voltage standards are becoming more liable to violation. Accordingly, intelligent voltage \\ management strategies are required to enable standards compliance without unduly increasing network \\ maintenance or infrastructure costs. The relationship between network power and voltages, however, is \\ complex, non-linear and interdependent, and thus is difficult to conceptualise and use for decision-making \\ and control purposes. This paper introduces a software toolkit that simplifies the development of voltage \\ management strategies by the application of sensitivity analysis. Sensitivity analysis reduces complex \\ network relationships to simple linear equations, and thus enables easy and comprehensive \\ conceptualisation of the effect of network modifications. Sensitivity data is obtained through a unique \\ 'perturb-and-observe' algorithm built on top of an open-source simulation package. The toolkit enables \\ the rapid development of sensitivity-driven network experimentation and is highly extensible. A number of \\ applications that demonstrate the usefulness of these techniques are presented, including the \\ development of a reactive power control algorithm for the mitigation of inverter- based, distributed \\ generation (DG)-induced voltage rise, and the verification of voltage-support-capacitor-bank placement for \\ a real Australian semi-rural network. Finally, this paper presents some suggestions for possible future \\ applications of data-driven, simulator-augmented sensitivity analysis techniques.
}

\section{Keywords}

network, distribution, mv, management, simplification, voltage, toolkit, analysis, sensitivity

\section{Disciplines}

Engineering | Science and Technology Studies

\section{Publication Details}

F. Tamp \& P. Ciufo, "A sensitivity analysis toolkit for the simplification of MV distribution network voltage management," IEEE Transactions on Smart Grid, vol. 5, (2) pp. 559-568, 2014. 


\title{
A Sensitivity Analysis Toolkit for the Simplification of MV Distribution Network Voltage Management
}

\author{
Fabian Tamp, Phil Ciufo Senior Member, IEEE
}

\begin{abstract}
As distribution networks become increasingly variable and generation becomes increasingly decentralised, voltage standards are becoming more liable to violation. Accordingly, intelligent voltage management strategies are required to enable standards compliance without unduly increasing network maintenance or infrastructure costs. The relationship between network power and voltages, however, is complex, non-linear and interdependent, and thus is difficult to conceptualise and use for decision-making and control purposes.

This paper introduces a software toolkit that simplifies the development of voltage management strategies by the application of sensitivity analysis. Sensitivity analysis reduces complex network $P-Q-|V|$ relationships to simple linear equations, and thus enables easy and comprehensive conceptualisation of the effect of network modifications. Sensitivity data is obtained through a unique 'perturb-and-observe' algorithm built on top of an open-source simulation package. The toolkit enables the rapid development of sensitivity-driven network experimentation and is highly extensible.

A number of applications that demonstrate the usefulness of these techniques are presented, including the development of a reactive power control algorithm for the mitigation of inverterbased, distributed generation (DG)-induced voltage rise, and the verification of voltage-support-capacitor-bank placement for a real Australian semi-rural network. Finally, this paper presents some suggestions for possible future applications of data-driven, simulator-augmented sensitivity analysis techniques.
\end{abstract}

Index Terms-Sensitivity Analysis, Power Distribution, Reactive Power Control, Load Flow, Smart Grids, Voltage Control.

\section{INTRODUCTION}

$\mathbf{I}$ $\mathrm{N}$ recent times, the prevalence of distributed generation (DG) and changing electricity consumption patterns have raised concerns about the maintenance of network voltage standards. In Australia, the widespread installation of photovoltaic generation has caused concern about the risk of overvoltage [1]. Australian Standard 60038-2000: Standard Voltages [2] specifies that the voltage experienced by the customer's point of supply should be $230 \mathrm{~V}$ with a maximum variation of $+10 \%$ and $-6 \%$. Historically, Australian standard voltages have been set to $240 \mathrm{~V}$, and thus for reasons of network consistency many Australian distribution network service providers (DNSPs) still set distribution (MV-LV) transformers to deliver a target $240 \mathrm{~V}$ to the customer [3]. DNSPs are discovering a need to rapidly develop and validate strategies for the management of changes in voltage.

Fabian Tamp is a recent graduate of the B. Engineering (Electrical) / B. Commerce degree from the University of Wollongong, Australia. Email: ft064@uowmail.edu.au

Phil Ciufo is a Senior Lecturer with the School of Electrical, Computer and Telecommunications Engineering University of Wollongong, Australia, and with the Australian Quality and Reliability Centre. Email: ppc@uow.edu.au

A preliminary version of this paper was submitted as a student-written paper to the IEEE/PES Student Prize Paper contest in December 2012.

The research in this paper was supported by the Australian Power Quality and Reliability Centre, http://www.elec.uow.edu.au/apqrc
The development of voltage management strategies, however, is a complex task - the relationship between network load and voltage magnitude is complex, multivariate and non-linear, and thus cause-and-effect relationships are not immediately visible when dealing with distribution network voltages. This makes network voltage relationships difficult to conceptualise, and thus significantly impedes the development of voltage management strategies. Often, the impact of new loads and generation on the network are assessed by building a simulation model, adding the new network element and manually examining the impact. This strategy works well enough when it is simply used to check that each network modification does not cause a violation of any existing voltage standards, but fails in situations where planning for future network modification is required. Often, the tools used for this kind of work lack the ability to readily provide the user with a quantifiable measure of relative effects. This tends to foster dependence upon a trial-and-error methodology to network modification, which in turn severely inhibits the conceptualisation, development, and communication of network voltage management strategies [4]. Traditional simulation tools are not conducive to data-driven decision-making, with decisionmakers often having to resort to the use of 'rules-of-thumb'.

Data-driven decision-making, however, is becoming increasingly necessary in an era where DNSPs are experiencing increased pressure to improve network efficiency and to make capital investment decisions with an extremely high level of accountability [5], [6]. Extensive network data is quickly becoming available, but the complexity and non-linearity of power systems makes the use of such network data difficult - the effect of changes in one part of the network upon other parts of the network cannot be easily quantified or expressed in a consistent fashion using traditional means, and thus cannot be cross-compared in a simple fashion.

Sensitivity analysis can help to overcome the difficulty that currently exists in making use of network data by projecting the complex equations that govern network voltages into a linear space. The equation for the change in voltage at a bus $\mathrm{Y}$ as a response to changes in $P$ and $Q$ at each network bus $\mathrm{X}$ is thus simplified to that given by (1) [7]:

$$
\Delta\left|V_{Y}\right| \approx \sum_{X}\left(\frac{\partial\left|V_{Y}\right|}{\partial P_{X}} \times \Delta P_{X}+\frac{\partial\left|V_{Y}\right|}{\partial Q_{X}} \times \Delta Q_{X}\right)
$$

This form of network equation enables the easy calculation of approximate changes in $|V|$ as a result of changes in $P$ and $Q$, enables buses to be ranked by their net effect on other buses, and enables the area of influence of a change in $P$ and $Q$ to be determined quickly and efficiently. In this form, the influence that network modifications have upon network voltages is easy to quantify, compare and conceptualise, 
greatly simplifying the development of coordinated voltage management strategies.

Sensitivity data arises through two main means: 'perturband-observe', that is, making a small change in network state and measuring the effect [8], and as a natural by-product of the Newton-Raphson load-flow algorithm [9], which is one of the most popular load-flow techniques for software simulation [10]. Despite the commonness of the Newton-Raphson load flow, sensitivity data is rarely accessible in modern network planning software. The ease of network conceptualisation offered by sensitivity analysis is thus largely unavailable to network engineers. There exists a need for a software solution that simplifies rapid prototyping and experimentation on network models, by making use of sensitivity techniques and providing for compatibility with existing network modelling schemes.

This paper introduces a new software toolkit that aims to resolve these difficulties inherent to the current simulator paradigm, by:

- Providing an infrastructure for easy prototyping and implementation of simulatory network experiments and control strategy development, in both off-line and on-line (real-time) contexts, and

- Providing tools for easy use of sensitivity analysis techniques within such experiments and control strategies.

The rest of this paper is structured as follows: Section II discusses the strengths and current applications of the sensitivity analysis paradigm for network analysis, coordination and management. Section III discusses the theory behind and assumptions intrinsic to sensitivity analysis, including the use of the Newton-Raphson load-flow and perturb-and-observe techniques for obtaining sensitivities. Section IV outlines the development of an electrical network experiment toolkit, including the practical implementation of a perturb-and-observebased sensitivity generation algorithm. Finally, Section V details some example applications of the developed methods, and outlines potential future extensions to the work.

\section{Strengths And Applications of the Sensitivity ANALYSIS TECHNIQUE}

\section{A. Strengths of the Sensitivity Analysis Technique}

Sensitivity analysis is advantageous compared to traditional simulation techniques because it transforms inter-bus $P-Q$ - $|V|$ relationships into a form that is easier to conceptualise and use for decision-making. The particular strengths of the technique lie in:

- Comparison of effect across a set of buses: the sensitivity coefficients $\frac{\partial\left|V_{Y}\right|}{\partial P_{X}}$ and $\frac{\partial\left|V_{Y}\right|}{\partial Q_{X}}$ provide direct numerical values that can be used to rank network buses in terms of their influence [7].

- Straightforward size determination of $P$ and $Q$ given a fixed $\Delta|V|$ : the maximum allowable load at a network bus to avoid a voltage violation can be quickly ascertained using the relationship [11]

$$
\Delta P_{X}=\frac{\Delta|V|_{\text {Target }}}{\frac{\partial\left|V_{Y}\right|}{\partial P_{X}}} .
$$

A similar relationship is available for sizing reactivepower-based voltage management solutions, such as voltage support capacitors [12]:

$$
\Delta Q_{X}=\frac{\Delta|V|_{\text {Target }}}{\frac{\partial\left|V_{Y}\right|}{\partial Q_{X}}} .
$$

Accordingly, sensitivity analysis is well suited to scenarios involving:

- Prioritisation of sensitive areas for voltage fluctuation management [9],

- 'Area-of-effect' determination of new power conversion or delivery equipment [4], and

- Placement decisions for loads, generation and voltage control devices in order to maximise or minimise their effect on system voltages [7], [11], [12].

A number of specific application examples from the existing literature are outlined in Section II-B, and a number of of proposed applications are discussed in Section V.

\section{B. Existing Applications of Sensitivity Analysis for Voltage Management}

Aghatehrani and Golnas [9] make use of same-bus sensitivity analysis as an integral part of a control strategy to minimise voltage fluctuations at network buses with PV installations. By calculating the ratio of $\frac{\partial\left|V_{X}\right|}{\partial P_{X}}$ to $\frac{\partial\left|V_{X}\right|}{\partial Q_{X}}$, an appropriate quantity of $Q$ can be determined for network injection or absorption in scenarios where $P$ fluctuates due to cloud shear and/or cover. Sensitivity analysis is particularly useful for distributed control solutions as computational requirements are low, making implementation of such strategies in real-world scenarios feasible.

Brenna, De Berardinis, Delli Carpini, et al. [7] derive a non-classical, low complexity formula for voltage magnitude sensitivities to changes in $P$ and $Q$ for radial mediumvoltage distribution networks. Sensitivities are calculated for a modified IEEE 13 Node Test Feeder, and then ranked and weighted in order to determine the 'most influential generator', that is, the generator on the network that has the greatest potential to affect network voltages by modulating real and reactive power output. A control scheme is developed that restores normal operating voltage in the event of a significant load disconnection (such as fault isolation) using this principle.

Sensitivity analysis has a further history of use in power systems for non-voltage-management applications. Acharya, Mahat, and Mithulananthan [11], for example, use a perturband-observe sensitivity approach to determine the optimal placement and sizing of distributed generation for the minimisation of network losses. Sensitivity information is also often used with respect to the determination and enhancement of network voltage stability [4], [12], [13].

\section{THEORETICAL BACKGROUND}

\section{A. Obtaining Sensitivity Data from the Newton-Raphson Load Flow}

Sensitivity data is readily obtained from the inverse of the standard Jacobian matrix $\boldsymbol{J}$ used for the calculation of network bus voltages under the Newton-Raphson load-flow technique [9]. The Newton-Raphson technique is an iterative load-flow approach, whereby the voltages in the circuit are set to some 
initial value and then repeatedly updated until the change in calculated values is smaller than a predetermined threshold. The update formula for the algorithm is given by (4):

$$
\left[\begin{array}{c}
\Delta \delta_{2} \\
\vdots \\
\Delta \delta_{n} \\
\hline \frac{\Delta\left|V_{2}\right|}{\left|V_{2}\right|} \\
\vdots \\
\frac{\Delta\left|V_{n}\right|}{\left|V_{n}\right|}
\end{array}\right]=\boldsymbol{J}^{-1}\left[\begin{array}{c}
\Delta P_{2} \\
\vdots \\
\Delta P_{n} \\
\hline \Delta Q_{2} \\
\vdots \\
\Delta Q_{n}
\end{array}\right] \text {, }
$$

where $\Delta \delta_{Y}$ and $\frac{\Delta\left|V_{Y}\right|}{\left|V_{Y}\right|}$ are the absolute change in phase and fractional change in voltage at bus $\mathrm{Y}$, respectively. In conventional load flow applications, $\Delta P_{X}$ and $\Delta Q_{X}$ are given by the difference between the specified complex bus power injected at bus $\mathrm{X}$ and values calculated based on computed bus voltages at the latest iteration and the nodal admittance matrix $\boldsymbol{Y}$. In this formulation, it is assumed that there is a reference bus of fixed voltage - in the case of the mediumvoltage distribution network, this would refer to the MV-side of the nearest upstream transformer.

Once the load-flow solution has converged, the Jacobian specifies the partial derivatives (that is, the sensitivities) of $P_{X}$ and $Q_{X}$ with respect to $\left|V_{Y}\right|$ and $\delta_{Y}$ as a function of current network state. An expression for $\boldsymbol{J}$ is given by (5) [10]:

$$
\boldsymbol{J}=\left[\begin{array}{ccc|ccc}
\frac{\partial P_{2}}{\partial \delta_{2}} & \cdots & \frac{\partial P_{2}}{\partial \delta_{n}} & \left|V_{2}\right| \frac{\partial P_{2}}{\partial\left|V_{2}\right|} & \ldots & \left|V_{n}\right| \frac{\partial P_{2}}{\partial\left|V_{n}\right|} \\
\vdots & \boldsymbol{J}_{\mathbf{1 1}} & \vdots & \vdots & \boldsymbol{J}_{\mathbf{1 2}} & \vdots \\
\frac{\partial P_{n}}{\partial \delta_{2}} & \cdots & \frac{\partial P_{n}}{\partial \delta_{n}} & \left|V_{2}\right| \frac{\partial P_{n}}{\partial\left|V_{2}\right|} & \cdots & \left|V_{n}\right| \frac{\partial P_{n}}{\partial\left|V_{n}\right|} \\
\hline \frac{\partial Q_{2}}{\partial \delta_{2}} & \cdots & \frac{\partial Q_{2}}{\partial \delta_{n}} & \left|V_{2}\right| \frac{\partial Q_{2}}{\partial\left|V_{2}\right|} & \cdots & \left|V_{n}\right| \frac{\partial Q_{2}}{\partial\left|V_{n}\right|} \\
\vdots & \boldsymbol{J}_{\mathbf{2 1}} & \vdots & \vdots & \boldsymbol{J}_{\mathbf{2 2}} & \vdots \\
\frac{\partial Q_{n}}{\partial \delta_{2}} & \cdots & \frac{\partial Q_{n}}{\partial \delta_{n}} & \left|V_{2}\right| \frac{\partial Q_{n}}{\partial\left|V_{2}\right|} & \cdots & \left|V_{n}\right| \frac{\partial Q_{n}}{\partial\left|V_{n}\right|}
\end{array}\right]
$$

Combining (4) and (5) yields the previously introduced simplified expression for an incremental change in voltage given by (1) [7]:

$$
\Delta\left|V_{Y}\right| \approx \sum_{X}\left(\frac{\partial\left|V_{Y}\right|}{\partial P_{X}} \times \Delta P_{X}+\frac{\partial\left|V_{Y}\right|}{\partial Q_{X}} \times \Delta Q_{X}\right),
$$

where $\Delta\left|V_{Y}\right|$ is the change in voltage magnitude at bus $\mathrm{Y}$, $\Delta P_{X}$ and $\Delta Q_{X}$ are the changes in $P$ and $Q$ at each network bus $X$ and $\frac{\partial\left|V_{Y}\right|}{\partial P_{X}}$ and $\frac{\partial\left|V_{Y}\right|}{\partial Q_{X}}$ are the sensitivities of $\left|V_{Y}\right|$ to changes in $P_{X}$ and $Q_{X}$ respectively.

This result is particularly useful, as it allows sensitivity information to be generated and validated as a by-product of a well-known load flow algorithm. Further, the sparse-matrix optimisations that can be applied to the Newton-Raphson load flow remain useful for the rapid calculation of network voltage sensitivities [12].

\section{B. Perturb-and-Observe Approach}

The method presented in Section III-A for obtaining sensitivities is convenient in situations where the Jacobian of the Newton-Raphson load-flow algorithm is accessible. This, however, is not always the case - the load-flow packages that use Newton-Raphson may not expose $\boldsymbol{J}$ to the user. Packages exist that use alternative techniques such as Gauss-Seidel or current injection methods for solving the load-flow problem. There are instances whereby the Newton-Raphson method will not work [14].

An alternative method traditionally used for obtaining sensitivities is 'perturb-and-observe', that is, the process of making small modifications and measuring the impact [8]. Whilst significantly less efficient when obtaining network sensitivities due to the need to re-compute the entire network state for a change on each bus, this strategy has the benefit of being loosely-coupled from the simulation approach - sensitivities are dependent only on having a working simulator. From a simulation perspective, this loose-coupling provides the benefit of allowing more resilient, application-specific or efficient simulation techniques to be selected. From an experiment and control algorithm development perspective, it allows existing models constructed with existing simulators to be used models do not need to be translated or re-constructed to fit a new system, so long as a software translator exists or can be built between the sensitivity generation layer and the simulator layer.

\section{Assumptions Intrinsic to Sensitivity Analysis}

The reader should note that the accuracy of sensitivity analysis approaches relies upon the following assumptions:

1) The set of $\Delta P_{X}$ and $\Delta Q_{X}$ must be small, due to the fact that the approximation formula projects a complex, non-linear system onto a linear system, and

2) The sensitivities are computed for the current state of the system, i.e., there must not be a significant accumulation of changes in $P$ and $Q$ across the network from the state in which the sensitivities were initially calculated.

Despite these inaccuracies, sensitivity analysis remains a useful tool for understanding the impact of network $P$ and $Q$ changes on network voltages, and for a first pass at decision making or algorithm development.

\section{DeVElopment of AN AnAlytical Network EXPERIMENT TOOLKIT}

\section{A. Purpose}

An open-source software toolkit named 'ElecNetKit' 1 (Electric Network Toolkit) was developed that resides as a software layer on top of existing simulator solutions. This toolkit places a heavy emphasis on simple and analytical examination of network state, and provides an infrastructure for modifying network state based upon these values. ElecNetKit ships with interfaces that ease obtaining and using network sensitivities, and particularly, inter-bus network voltage sensitivities to changes in $P$ and $Q$.

ElecNetKit was designed in a fashion that enables highlevel exploration and understanding of network relationships. With simplicity as a design goal, ElecNetKit uses a completely modular system by which simulation experiments can quickly be written, executed, and validated, with results readily able to be transformed and graphed. A desire to make best use

\footnotetext{
${ }^{1}$ The ElecNetKit project is available for examination and use at http://github.com/ElecNetKit/ElecNetKit.
} 
of the vast data available within a smart grid context dictates interoperability with external data sources as another key goal.

\section{B. Software Implementation}

Presently, ElecNetKit is capable of using OpenDSS, an open-source distribution systems simulator package developed by the Electric Power Research Institute (EPRI), as a simulator backend. OpenDSS is supplied with both a text-driven interface and a programmable COM (Component Object Model) interface, over which OpenDSS can be used as a software library. Many simulators have programmable interfaces, but OpenDSS is relatively unique in that software interoperability and extensibility is a high-priority development goal [15]. The supplied text interface, however, is somewhat limited for the purposes of performing computationally complex experiments, such as those required for control strategy development. Whilst the COM programming interface exposes all required functionality, it can be quite difficult to use, and does not enable network relationships or characteristics to be explored in a high-level way.

The electric network toolkit is a higher-level abstraction of the basic functionality exposed by OpenDSS, using OpenDSS models as a starting point, and enabling programmatic experimentation and analysis of these distribution network models in accordance with the goals described in Section IV-A. As a software library, ElecNetKit requires knowledge of a programming language for use, but has the advantages of flexibility and interoperability with any data that the host computer can process.

The basic ElecNetKit architecture is illustrated in Fig. 1. Network Experiment modules are written by the user in a simple plug-in format that takes an existing network model as an input and issues commands to modify the network. Results Processor modules are also written by the user and hook into the experimentation process, obtaining network models before and after the experiment has occurred and processing results arbitrarily. A number of sample Network Graphing modules are supplied with ElecNetKit, and can be customised as required.

\section{Implementation of a Perturb-and-Observe Sensitivity Gen- erator}

A perturb-and-observe network algorithm, as documented in Algorithm 1, is used to generate sensitivities from electrical network models. The algorithm is implemented on top of the ElecNetKit base layer. The loose-coupling intrinsic to the perturb-and-observe approach makes it a natural choice given the aims and architecture of ElecNetKit.

A Network Experiment module is used to add a small generator into the network on a specified bus $X$. This module works in accord with a simple Results Processor, which subtracts pre-experiment network bus voltage magnitude and phase from post-experiment network bus voltage magnitude and phase. The Results Processor also locates the inserted generator within the modified network topology and uses the bus voltage differentials together with the generation quantity to compute the sensitivities $\frac{\partial|V|}{\partial P_{X}}$ and $\frac{\partial \delta}{\partial P_{X}}$ for each network bus. The entire experimentation system is then run in a loop, choosing a different source bus on each iteration, and setting

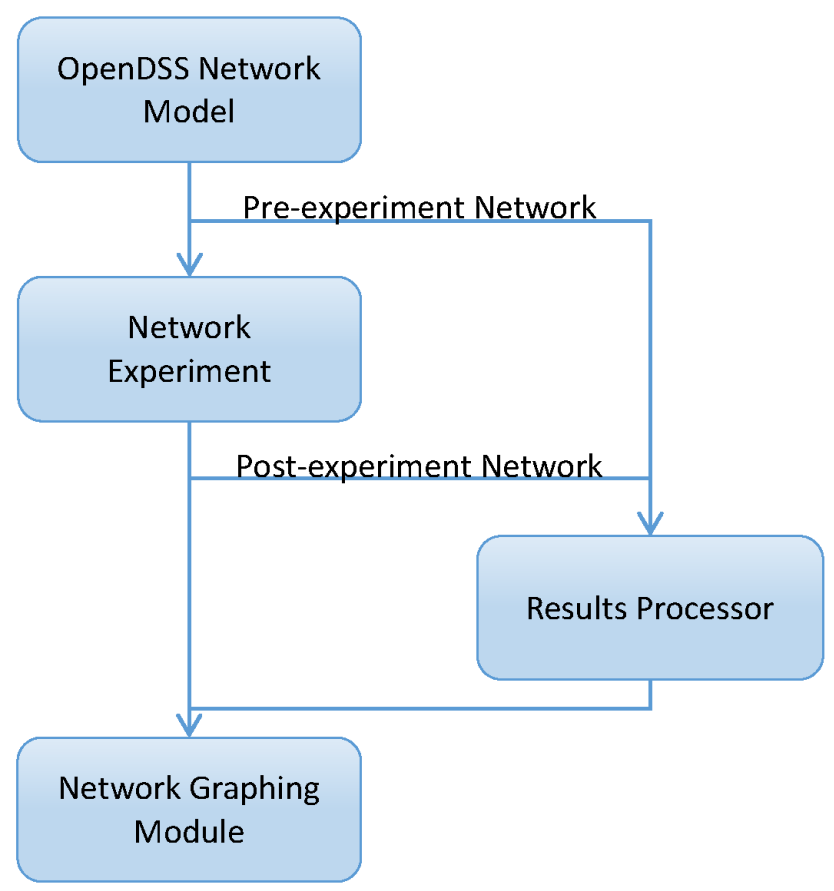

Fig. 1. Electric Network Toolkit Architecture

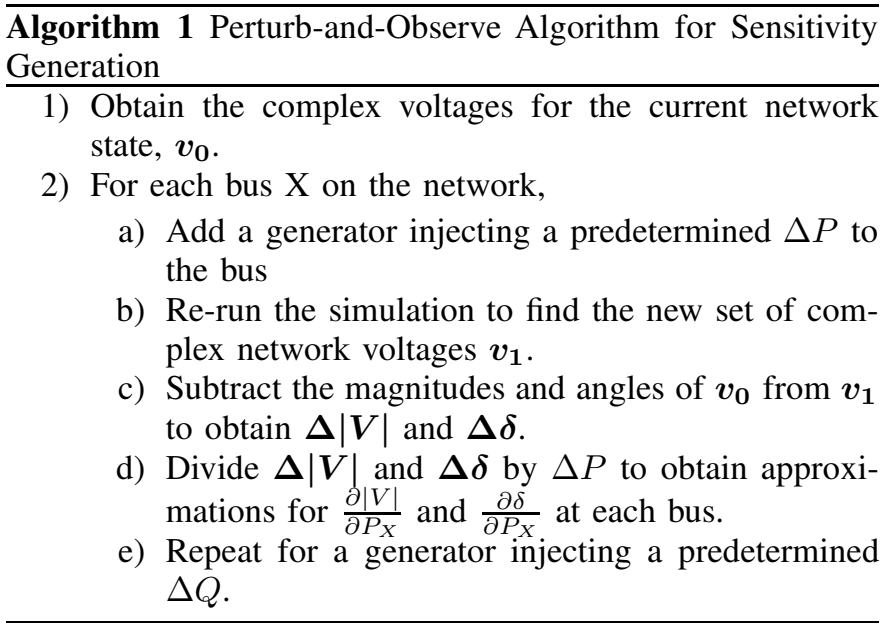

a generator to inject a small quantity of $P$ and $Q$ in different experiments.

There is an important performance consideration here the number of simulations required to calculate sensitivities across the network is proportional to the number of network buses. The performance further depends upon the number of simulator iterations requires for convergence, and thus also depends upon the simulator in use, the specified simulator accuracy and the characteristics of the specific network model under investigation.

The architecture of ElecNetKit and its use of a complete simulator package at its core lends itself to high extensibility. As such, the specific example of voltage sensitivity to $P$ and $Q$ could readily be modified to determine the sensitivity of network losses to changes in $P$ and $Q$, the voltage sensitivity to phase imbalance, or the harmonic strength sensitivity to changes in network loading and generation. These potential future applications are discussed further in Section V-C. 


\section{Error Considerations and Perturbation Quantity Selection}

As discussed in Section III-C, it is important for $\Delta P$ and $\Delta Q$ to be chosen to be 'sufficiently small' such that the approximations $\frac{\Delta|V|}{\Delta P} \approx \frac{\partial|V|}{\partial P}$ and $\frac{\Delta|V|}{\Delta Q} \approx \frac{\partial|V|}{\partial Q}$ (together referred to as the "tangency approximation' henceforth throughout this paper) hold. The values for $\Delta P$ and $\Delta Q$ for which these approximations are valid, however, differ for every network, and so some level of engineering intuition has to be used in order to determine an appropriate value. On the other hand, $\Delta P$ and $\Delta Q$ need to be sufficiently large such that the limit of accuracy of the simulated voltages is not significant compared to the change in the voltage induced by the perturbation. This tradeoff is made more difficult by the fact that the error due to the tangency approximation is not directly measurable without undertaking the Newton-Raphson load flow and obtaining the Jacobian. The error and fractional error in sensitivity due to the limit of accuracy (LOA) of the simulated voltages can be found by incorporating the LOA into the perturb-and-observe sensitivity equation, in which $V_{0}$ and $V_{1}$ represent the bus voltage before and after perturbation, respectively:

$$
\begin{aligned}
\frac{\partial|V|}{\partial P} & \approx \frac{\Delta|V|}{\Delta P} \\
& =\frac{\left(\left|V_{1}\right| \pm \mathrm{LOA}\right)-\left(\left|V_{0}\right| \pm \mathrm{LOA}\right)}{\Delta P} \\
& =\frac{\left|V_{1}\right|-\left|V_{0}\right|}{\Delta P} \pm \frac{2 \times \mathrm{LOA}}{\Delta P}
\end{aligned}
$$

Thus, the maximum error in sensitivity due to the limit of accuracy of the simulated voltages is given by (6):

$$
\varepsilon_{\max }=\frac{2 \times \mathrm{LOA}}{\Delta P}
$$

Dividing both sides of (6) by the sensitivity $\frac{\partial|V|}{\partial P}$ gives the fractional error due to the limit of accuracy, as expressed by (7):

$$
\begin{aligned}
\frac{\varepsilon_{\max }}{\frac{\partial|V|}{\partial P}} & =\frac{2 \times \mathrm{LOA}}{\Delta P \frac{\partial|V|}{\partial P}} \\
& \approx \frac{2 \times \mathrm{LOA}}{\Delta P \frac{\Delta|V|}{\Delta P}} \\
& =\frac{2 \times \mathrm{LOA}}{\Delta|V|}
\end{aligned}
$$

The same logic applies to the maximum error and fractional error for the sensitivity $\frac{\partial|V|}{\partial Q}$.

An important consequence of this result is that the error due to the limit of accuracy will be larger on buses with less variable voltages, such as those close to the bulk supply point. At such buses, however, the sensitivity to and influence upon other network buses are typically smaller. Consequently, these buses are of less significance for voltage management strategies, and thus the increased error at these buses would usually have a minimal effect on application of these techniques. Nevertheless, for such buses, the error could be managed by either:

- specifying a higher limit of accuracy for simulator convergence, and/or

- scaling the magnitude of $\Delta P$ and $\Delta Q$ to induce a larger change in voltage.
Adopting either of these error minimisation strategies is not without problems - narrowing the simulator limit of accuracy may vastly increase solution convergence time, or may cause the simulation to never converge at all. Increasing the magnitude of $\Delta P$ and $\Delta Q$ may increase the error due to the tangency approximation to an unreasonable level.

When working with distribution networks, a sensible strategy is to set $\Delta P$ and $\Delta Q$ to a percentage of the average load magnitude. For a simple test network (detailed in Section IV-E1), values of between $0.5 \%$ and $2 \%$ were found to yield acceptable results for a simulator limit of accuracy of $1 \times 10^{-7}$ p.u.

\section{E. Validation of Results}

1) Manual Calculation of Expected Sensitivities: In order to validate the developed method, a Newton-Raphson loadflow algorithm was written in MATLAB. This custom loadflow algorithm was verified by comparing the obtained outputs from the load-flow against the test network illustrated in Fig. 2. The test network is a simple, hypothetical, $230 \mathrm{kV}$ transmission network, with a total loading of $570 \mathrm{MW}$ and 309.86 Mvar, and was chosen for the reason that it is simple enough such that calculations are hand-verifiable in the case of discrepancies. After the custom MATLAB load-flow had converged within a limit of accuracy of $10^{-7}$ p.u., the Jacobian $\boldsymbol{J}$ was extracted and inverted to obtain the matrix given in (8) [12]:
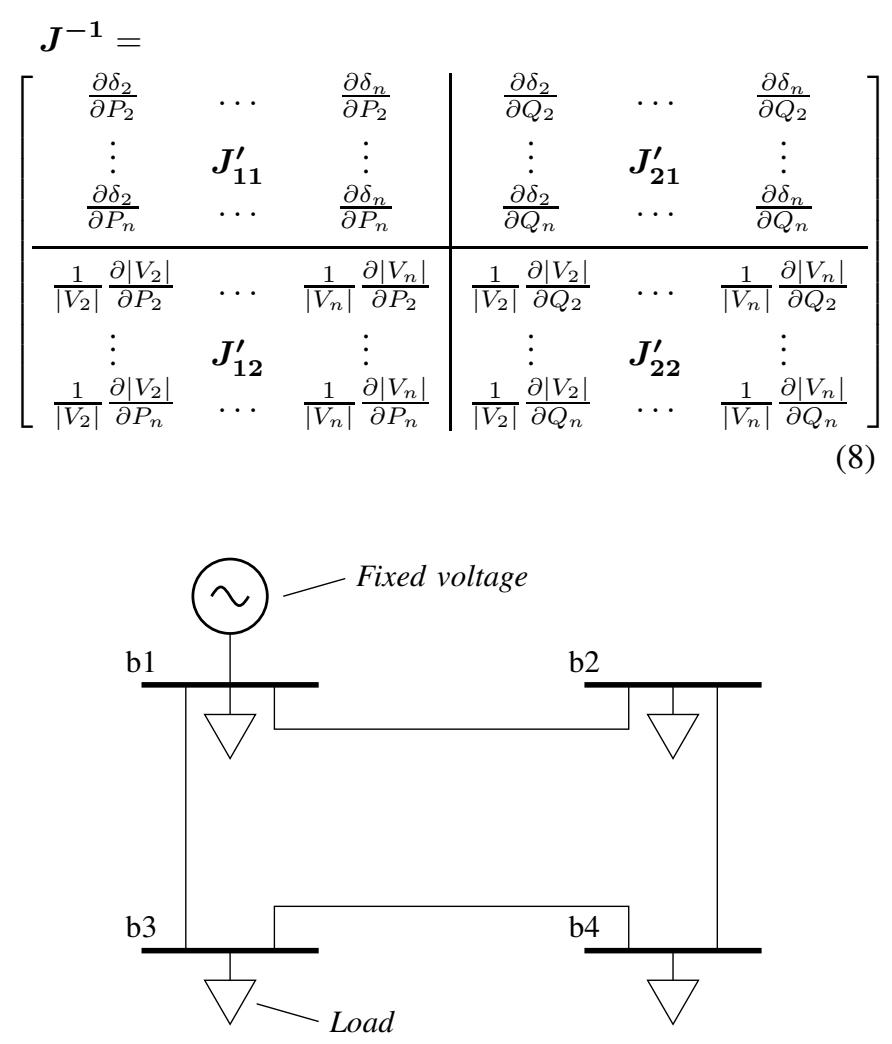

Fig. 2. Test Network Topology for Manual Validation, adapted from Grainger and Stevenson [10]

The values in $\boldsymbol{J}_{\mathbf{2} 1}^{\prime}$ and $\boldsymbol{J}_{\mathbf{2} 2}^{\prime}$ were then multiplied by the respective voltages in order to cancel the $\frac{1}{|V|}$ terms, and thus to obtain the reference set of voltage magnitude $|V|$ 
and voltage angle $\delta$ sensitivities to changes in $P$ and $Q$. At this point, the obtained sensitivities were in per-unit form and thus were converted into absolute values for comparison with results generated from the electric network toolkit. Voltage magnitude sensitivities were multiplied by $\frac{V_{\text {base }}}{S_{\text {base }}}$, and voltage angle sensitivities were multiplied by $\frac{1}{S_{\text {base }}}$.

The MATLAB load-flow algorithm was then used to calculate sensitivities using the perturb-and-observe approach outlined in Section III-B. These sensitivities were then compared to the reference set.

Finally, the test network was implemented in OpenDSS, and the perturb-and-observe algorithm was implemented using the electric network toolkit. Statistics for the error between the sensitivities obtained from the ElecNetKit perturb-andobserve approach and the reference set sensitivities are given in Table I, using the results from the inverse Jacobian as the baseline values. As expected, there was negligible (less than $10^{-6}$ ) difference between all sensitivities obtained with the MATLAB-implemented perturb-and-observe strategy and the electric network toolkit results. The error indicated in Table I, then, is purely due to the tangency approximation, that is, due to the linearisation of a non-linear system.

TABLE I

ERROR STATISTICS FOR EXAMPLE NETWORK VOLTAGE SENSITIVITIES AS CALCULATED BY THE ELECTRIC NETWORK TOOLKIT, COMPARED TO THE INVERTED JACOBIAN VIA MATLAB, USING $\Delta P, \Delta Q=2 \%$ OF AVERAGE LOAD MAGNITUDE

\begin{tabular}{ccc}
\hline Sensitivity & Median Error & Maximum Error \\
\hline$\frac{d|V|}{d P}$ & $0.342 \%$ & $0.452 \%$ \\
$\frac{d|V|}{d Q}$ & $0.188 \%$ & $0.240 \%$ \\
$\frac{d \delta}{d P}$ & $0.061 \%$ & $0.105 \%$ \\
$\frac{d \delta}{d Q}$ & $0.602 \%$ & $2.368 \%$ \\
\hline
\end{tabular}

2) Validation against Sensitivities Obtained using Another Simulator: As an additional means of verification, a balanced version of the $4.16 \mathrm{kV}$ IEEE 13-bus network [16] was obtained as a DigSILENT PowerFactory model. This modified IEEE 13-bus network has a total loading of 3.8 MW and 2.4 Mvar, maintains voltages between 1.0 p.u. and 0.95 p.u, and is illustrated in Fig. 3. The balanced IEEE 13-bus network was then implemented as an OpenDSS model. Network sensitivities were then calculated using both PowerFactory's 'Load Flow Sensitivities' feature and the ElecNetKit perturb-andobserve system. Statistics for the error between the sensitivities calculated by PowerFactory and the electric network toolkit are given in Table II. The errors obtained were in all cases smaller than $0.3 \%$, even including the error due to the slight differences in the way that the two simulator back-ends model transformer impedances. These results, along with those of of Section IV-E1, indicate that the sensitivities obtained are accurate enough for use in most practical situations.

\section{CASe Studies}

A. A Reactive Power Management Strategy to Compensate for DG-induced Voltage Rise

1) Algorithm Design: A reactive power coordination algorithm was developed to counteract DG-induced voltage rise at the MV level as an example application of the sensitivity

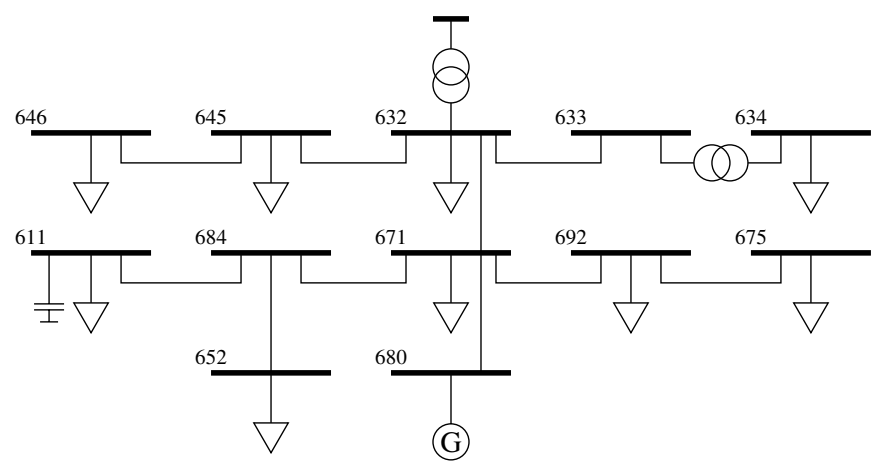

Fig. 3. Balanced IEEE 13-bus network adapted from Jayawardena et. al. [16]

TABLE II

ERROR STATISTICS FOR MODIFIED IEEE 13-BUS NETWORK VOLTAGE SENSITIVITIES AS CALCULATED BY THE ELECTRIC NETWORK TOOLKIT, COMPARED TO DIGSILENT POWERFACTORY RESULTS, USING $\Delta P, \Delta Q=1 \%$ OF AVERAGE LOAD MAGNITUDE

\begin{tabular}{ccc}
\hline Sensitivity & Median Error & Maximum Error \\
\hline$\frac{d|V|}{d P}$ & $0.078 \%$ & $0.090 \%$ \\
$\frac{d|V|}{d Q}$ & $0.254 \%$ & $0.273 \%$ \\
$\frac{d \delta}{d P}$ & $0.236 \%$ & $0.267 \%$ \\
$\frac{d \delta}{d Q}$ & $0.067 \%$ & $0.101 \%$ \\
\hline
\end{tabular}

analysis toolkit. This algorithm operates by coordinating the reactive power absorption (that is, the injection of a negative quantity of reactive power) across multiple generators to reduce the voltage rise induced by the real power output of the distributed generators. This algorithm utilises a 'most influential generator' strategy similar to that outlined by Brenna, De Berardinis, Delli Carpini, et al. [7], but optimises reactive power levels across a number of generators to better meet target values. Whilst generators have been exclusively used as control points for this specific example, the algorithm is not bound to them - in theory, any controllable reactive powersource could be utilised. The sensitivities used in the algorithm are calculated for the network state prior to algorithmic changes using the perturb-and-observe approach outlined in Section IV-C. The steps of the algorithm are listed in detail in Algorithm 2.

2) Algorithm Results: The algorithm was tested for a hypothetical scenario on a model of a real, semi-rural network obtained from an Australian DNSP. The model is of an $11 \mathrm{kV}$ network, that consists of six feeders and approximately 250 buses, each corresponding to an MV-LV distribution substation. The network as a whole has a maximum yearly demand of approximately 14.3 MW. A generator was added to each of a group of $20 \mathrm{MV}-\mathrm{LV}$ distribution substations toward the end of a long feeder, as indicated in Fig. 4. The generators were set to output a constant $\mathrm{kW}$ equivalent to the maximum demand of the attached distribution substation. Historical network load data was obtained, and the substation loadings were set to their load at the time when total network loading was at a minimum, representing the worst-case scenario for voltage rise on the network [1]. The network loading at this time was $23 \%$ of maximum demand. The maximum percentage of reactive power absorption allowable $Q_{\max } \%$ was varied between $5 \%$ and 


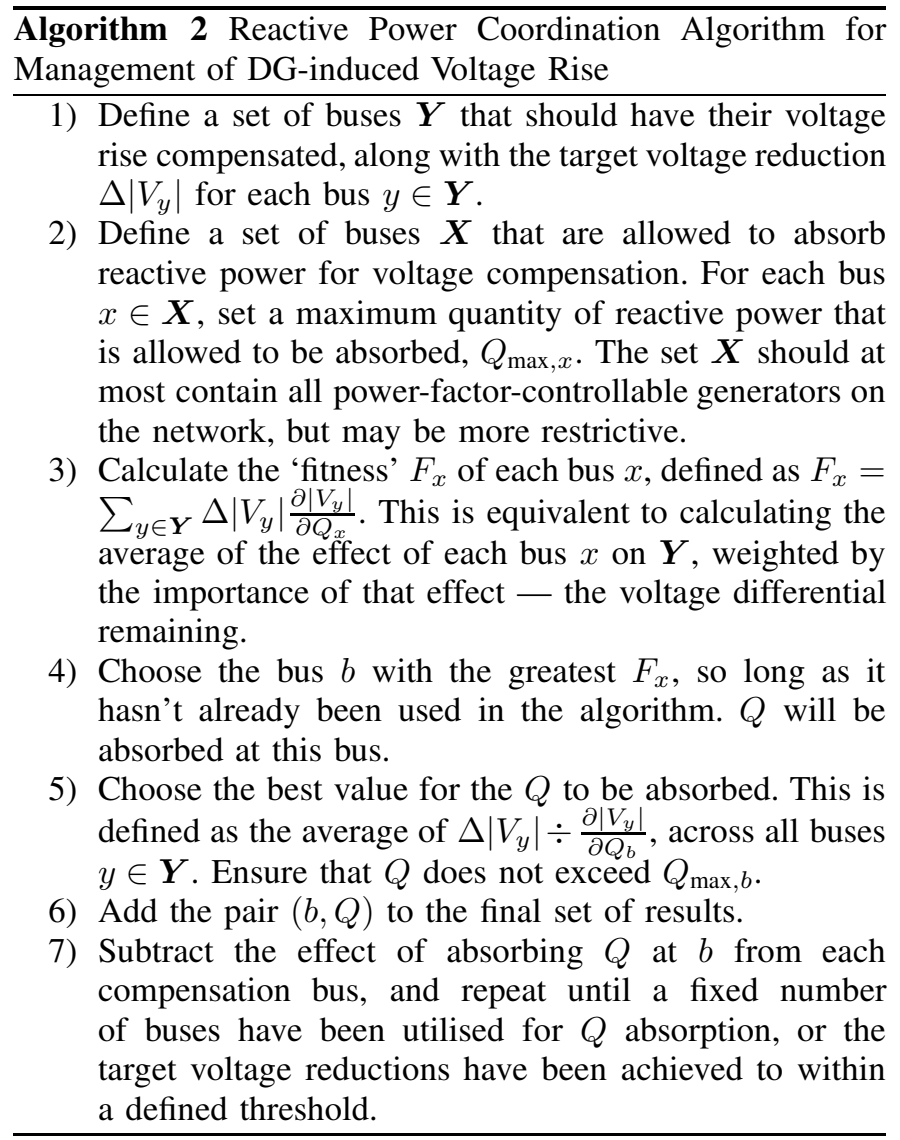

$130 \%$ of generator $\mathrm{kW}$ output, and the effect of the voltage compensation algorithm was measured.

Performance of the reactive power compensation algorithm varied with the maximum allowable reactive power absorption. For the test scenario, compensation of up to $90 \%$ of the DGinduced voltage rise was attainable, when generators were allowed to absorb as much as $105 \%$ of their real power output in reactive power. As discussed in Section V-A3, however, reactive power absorption of this magnitude may be impractical, or restricted by regulation. For a more sensible maximum absorption level of $60 \%$ of real power output, the DG-induced voltage rise can still be compensated by up to $48 \%$, for an average generator power factor of 0.86 .

Fig. 5 illustrates the performance of the algorithm, as measured by the average reduction in DG-induced voltage rise across affected buses, for various values of $Q_{\max \%}$.

3) Implications of Algorithm Implementation: Reactive power-based voltage management algorithms are best suited to inverter-based DG installations, due to the ability of many inverters to have an operating phase set. Globally, however, there are restrictions regarding the level of reactive power absorption or injection allowed into the network [17], and this may restrict the level of $Q$ absorption allowable.

An additional technical aspect of such a voltage control methodology is that the use of reactive power absorption for voltage control requires inverters to have capacity oversized in proportion to $\sqrt{1+Q_{\max }^{2}}$. For the aforementioned case of $Q_{\max \%}=60 \%=0.6$, the average generator would need to be oversized by approximately $17 \%$.

The reader should note that the additional reactive power

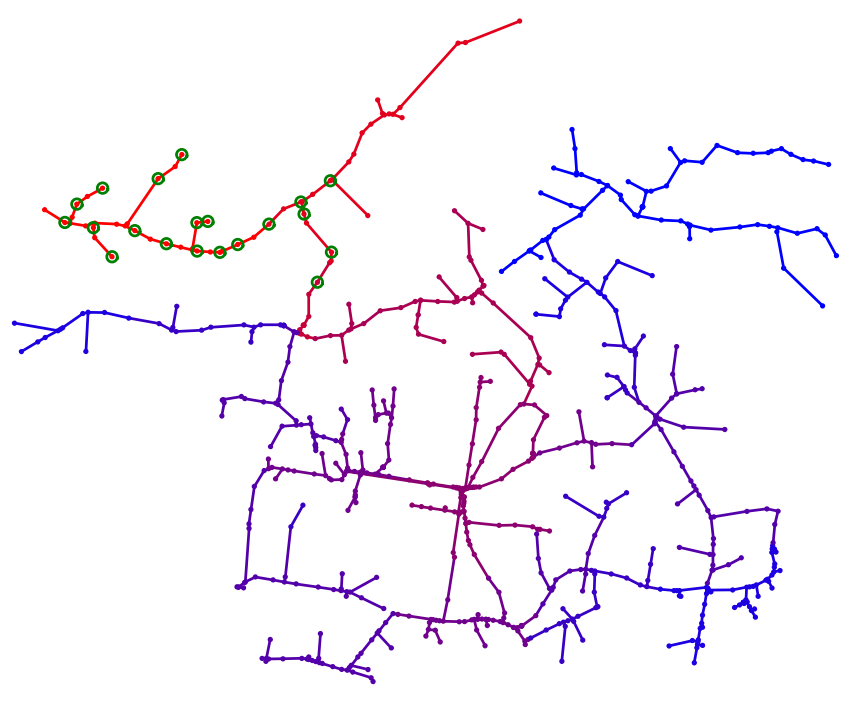

Fig. 4. Positioning of generators for algorithm test scenario. Generators are indicated by small rings on the appropriate substations.

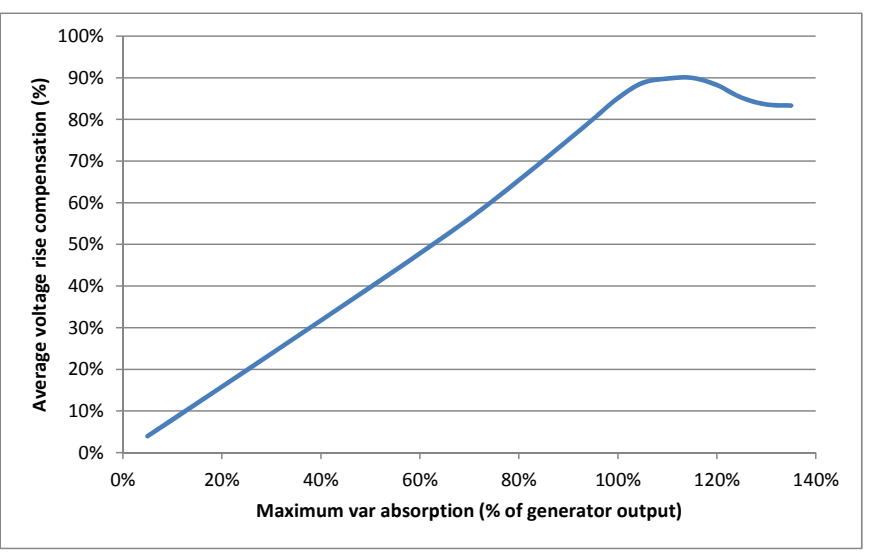

Fig. 5. Algorithm performance for varying maximum allowable reactive power absorption.

drawn by this system would incur additional network losses compared to scenarios in which all generators operate at unity power factor. These losses would need to be balanced against the generation capacity gained by using such a method, and factored in to the cost of generator connection. For the example case of $Q_{\max \%}=60 \%$, total network losses were found to increase by $5 \%$ of the installed generation capacity.

The implementation of such a system would draw a significant out-of-phase current. Under heavily loaded conditions, drawing such a current could lead to failure of network equipment. The DG-induced voltage rise, however, would only cause a violation of Australian voltage standards in low-load situations. The use of such a mitigation strategy would thus be unwarranted at times when the additional current draw induced could pose a problem.

4) Further Work: The reactive power control example presented in this subsection has been confined to the case of a single static network, at the lowest experienced network loading. Examining the adjustment of such an algorithm to fluctuating network loading conditions would be worthwhile, as well as examining the application of this technique to different network topologies, such as grid-structured (non- 
radial) networks. The process of converting the algorithm to run on series or real-time data would only involve supplying alternative data to the model, and running the algorithm continuously.

Finally, the case of using reactive power injection for voltage support could be considered, under conditions of heavy loading and low availability of generation. This would be of particular relevance to the Australian context, and would be a likely occurrence in the evening, when demand is at a maximum [18], and solar irradiation is low.

\section{B. Verification of Capacitor Siting and Sizing for Voltage Support}

On another feeder on the MV network used in Section V-A, increasing feeder loads were causing undervoltage problems on high-load days. As such, the DNSP had installed a 600 kvar voltage support capacitor bank on the feeder. The developed sensitivity analysis toolkit was used to verify the capacitor rating chosen by the DNSP, using the relationship given in (3). A network graph of the sensitivity of the end-of-feeder voltage to the changes in $Q$ at each bus is presented in Fig. 6 . The sensitivity of the end-of-feeder voltage to the bus marked C was:

$$
\frac{\partial\left|V_{\text {end }}\right|}{\partial Q_{C}}=0.14587 \mathrm{~V}_{\mathrm{LN}} / \mathrm{kVA}_{3 \phi},
$$

and thus

$$
\begin{aligned}
\Delta\left|V_{\text {end }}\right| & =\frac{\partial\left|V_{\text {end }}\right|}{\partial Q_{C}} \times Q_{C} \\
& =0.14587 \frac{\mathrm{V}_{\mathrm{LN}}}{\mathrm{kVA}_{3 \phi}} \times 600 \mathrm{kVA}_{3 \phi} \\
& =87.522 \mathrm{~V}_{\mathrm{LN}} \\
& =0.0138 \text { p.u. }
\end{aligned}
$$

This value agrees reasonably closely with the target voltage rise value of 0.0113 p.u. determined by the DNSP.

\section{Other Potential Applications}

Further applications of the techniques developed here could include:

- Placement of distributed generation and storage for the purposes of voltage management. Sensitivity analysis provides a straightforward first pass for examining the impact of proposed generation and storage, and determining alternative locations at which such technologies could minimise voltage rise or provide enhanced voltage support or more effective voltage control.

- Placement and control strategies of reactive power management devices, including static synchronous compensators (DSTATCOMs) and static var compensators (SVCs). Such devices are becoming increasingly popular in embedded generation scenarios [19], and are popularly being used for voltage management and fault-ridethrough, especially in microgrids [4]. These devices could be easily placed for maximum effect using sensitivity analysis techniques, and integrated control schemes could further prioritise data from measurement locations with known higher sensitivities.

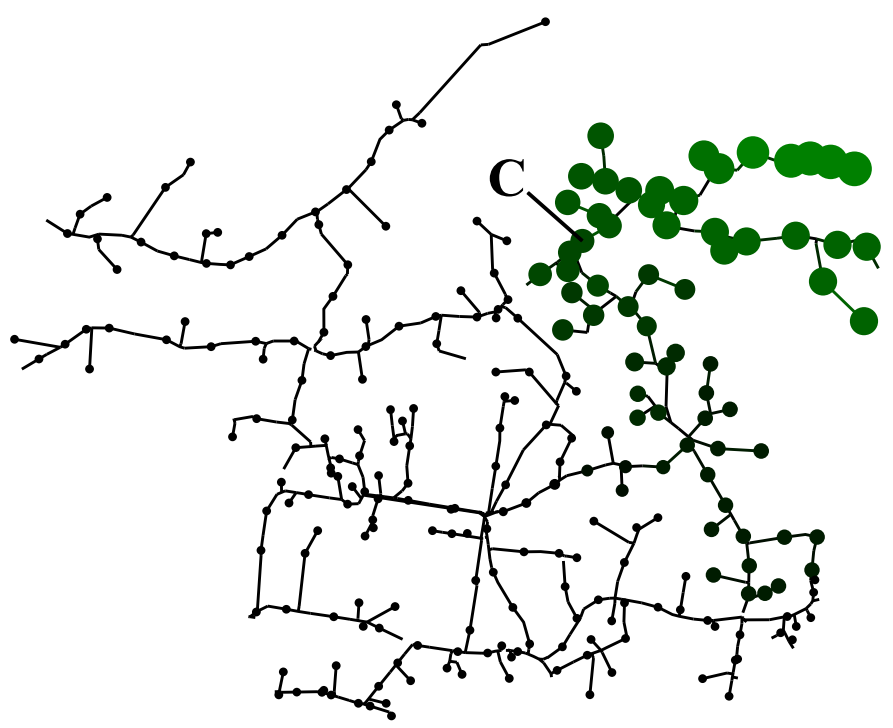

Fig. 6. Sensitivity of the voltage magnitude at the end of the feeder to $Q$ at each network bus. A larger spot indicates that a bus has a greater effect upon the voltage at the end of the feeder. $\mathbf{C}$ represents the capacitor bank location chosen by the DNSP.

Further, the sensitivity analysis techniques used within ElecNetKit are highly extensible, as mentioned in Sections III-B and IV. The generality of the perturb-and-observe sensitivity generation algorithm utilised lends itself to application to other, non-voltage sensitivity problems, such as the sensitivity of total network losses to changes in $P$ and $Q$ at specific buses, or sensitivity of the network to harmonic injection. Such customised sensitivity data could not normally be obtained through the application of the Newton-Raphson loadflow algorithm, and thus the perturb-and-observe method has particular strength in these domains.

Finally, the sensitivity analysis techniques presented throughout this paper could be applied for real-time network control scenarios by simply switching the simulator backend with one that targets network hardware. This use-case, however, warrants an additional consideration - sensitivities do not remain valid for significant changes in network loading, and thus need to be re-calculated periodically. The quantity of simulations required by the perturb-and-observe method means that processing time may become significant for large networks. This can, however, be mitigated in part through use of modern hardware and multi-threading approaches though the availability of multi-threading within the toolkit depends upon the multi-threading compatibility of the underlying simulator $^{2}$. For medium-voltage networks of equivalent size to the network used in Section V-A, sensitivity update times would easily be able to keep up with significant deviations in network state when processing is undertaken on commodity server hardware. For larger, more complex, or more dynamic networks, prioritisation strategies could be undertaken to speed calculation for practical real-time algorithm implementation, such as only calculating sensitivities for buses at which some

\footnotetext{
${ }^{2}$ The only current simulator implementation, OpenDSS, does not, at time of writing, natively support multi-threading [20]. There are strategies for working within this constraint, though they add significantly to the complexity of the use of ElecNetKit.
} 
aspect of the network can be controlled.

Currently, most Australian DNSPs take network loading measurements every ten to fifteen minutes, which is within the same approximate range as the processing time required for the $250 \mathrm{MV}-\mathrm{LV}$ substation distribution network model used in Section IV-B. If sensitivity data were to be updated to remain accurate to the current measured network state for real-time control scenarios, it could be worthwhile to consider performance improvements to the system proposed.

\section{CONCLUSIONS}

Intelligent voltage management strategies will be increasingly required as electrical networks evolve. This paper has presented a toolkit designed to ease development of such strategies through use of sensitivity analysis techniques, using an approach that emphasises ease of experimentation. It is hoped that the tools developed provide a foundation for enabling both members of the academic community and industry to rapidly experiment upon existing, real-world network models, in a fashion unrestricted by the current analysis paradigms used by most modern distribution simulation software.

The developed techniques have been applied to the problem of mitigating inverter-based-DG-induced voltage rise through reactive power compensation, achieving strong results at various allowable levels of reactive power absorption. They have also been applied to the case of capacitor placement and sizing for voltage support.

The proposed techniques include an open-source, extensible perturb-and-observe approach to the generation of network sensitivity information, which could readily be adapted to provide useful information on network loss sensitivity to changes in and placement of $P$ and $Q$, voltage unbalance and harmonic analysis. Open-source tools are an important resource for academics, researchers and practitioners, providing freedom to explore different methods of analysing and visualising network performance without being tied to a single, proprietary platform, and enabling collaboration.

\section{REFERENCES}

[1] M. A. Mahmud, M. J. Hossain, and H. R. Pota, "Worst case voltage variation on microgrid," in Smart Power Grids 2011, ser. Power Systems, A. Keyhani and M. Marwali, Eds. Springer Berlin Heidelberg, Jan. 2012, pp. 305-318. [Online]. Available: http://link.springer.com/chapter/10.1007/978-3-642-21578-0_10

[2] Standards Australia, "Australian standard 600382000: Standard voltages," 2000. [Online]. Available: http://infostore. saiglobal.com/store/Details.aspx?ProductID=321155

[3] C. Halliday, "When voltage varies," Electrical Connection, no. Winter 2012, Jul. 2012. [Online]. Available: http://www.elect.com.au/Attachments/EC-2-2012\%20Voltage.pdf

[4] J. D. Glover, M. S. Sarma, and T. Overbye, Power System Analysis and Design, Fifth Edition, 5th ed. Cengage Learning, Jan. 2011.

[5] C. Vespi and J. Hazen, "Big data, bigger opportunities: Plans and preparedness for the data deluge," Oracle, Tech. Rep., Jul. 2012. [Online]. Available: http://www.oracle.com/ocom/groups/public/@ocom/documents/webconte

[6] T. Wood, A. Hunter, P. Venkataraman, M. O'Toole, and L. Carter, "Putting the customer back in front: How to make electricity cheaper," Grattan Institute, Tech. Rep. 2012-09, Dec. 2012. [Online]. Available: http://grattan.edu.au/static/files/assets/7d0a806e/178_energy_putting_the_cu

[7] M. Brenna, E. De Berardinis, L. Delli Carpini, F. Foiadelli, P. Paulon, P. Petroni, G. Sapienza, G. Scrosati, and D. Zaninelli, "Automatic distributed voltage control algorithm in smart grids applications," IEEE Transactions on Smart Grid, vol. 4, no. 2, pp. 877-885, 2013.

[8] K. Christakou, J.-Y. Le Boudec, M. Paolone, and D.-C. Tomozei, "Efficient Computation of Sensitivity Coefficients of Node Voltages and Line Currents in Unbalanced Radial Electrical Distribution Networks," IEEE Transactions on Smart Grid, 2013.

[9] R. Aghatehrani and A. Golnas, "Reactive power control of photovoltaic systems based on the voltage sensitivity analysis," in 2012 IEEE Power and Energy Society General Meeting, Jul. 2012, pp. 1 -5.

[10] J. Grainger and W. Stevenson, Jr, Power System Analysis, 1st ed. McGraw-Hill Science/Engineering/Math, Jan. 1994.

[11] N. Acharya, P. Mahat, and N. Mithulananthan, "An analytical approach for DG allocation in primary distribution network," International Journal of Electrical Power \& Energy Systems, vol. 28, no. 10, p. 669678, 2006.

[12] M. Begovic and A. Phadke, "Control of voltage stability using sensitivity analysis," IEEE Transactions on Power Systems, vol. 7, no. 1, pp. 114 -123 , Feb. 1992.

[13] R. Monteiro Pereira, C. Machado Ferreira, and F. Maciel Barbosa, "Dynamic voltage stability assessment of an electric power system using trajectory sensitivity analysis," in PowerTech, 2009 IEEE Bucharest, Jul. 2009, pp. $1-6$.

[14] M. H. Haque, "A general load flow method for distribution systems," Electric Power Systems Research, vol. 54, pp. 47-54, 2000.

[15] R. C. Dugan, Reference Guide: The Open Distribution System Simulator (OpenDSS). Electric Power Research Institute, Inc., Mar. 2012. [Online]. Available: http://sourceforge.net/projects/electricdss/files/

[16] A. Jayawardena, L. Meegahapola, S. Perera, and D. Robinson, "Dynamic characteristics of a hybrid microgrid with inverter and noninverter interfaced renewable energy sources: A case study," Faculty of Engineering and Information Sciences - Papers, pp. 1-6, Jan. 2012. [Online]. Available: http://ro.uow.edu.au/eispapers/265

[17] Standards Australia, "Australian standard 4777.2-2005: Grid connection of energy systems via inverters — inverter requirements," 2005. [Online]. Available: http://infostore.saiglobal.com/store/Details.aspx?ProductID=318072

[18] A. Nizar, Z. Dong, and J. Zhao, "Load profiling and data mining techniques in electricity deregulated market," in IEEE Power Engineering Society General Meeting, 2006, 2006, p. 7 pp.

[19] M. J. Hossain, H. R. Pota, and M. A. Mahmud, "Decentralized STATCOM/ESS control for wind generators," in Smart Power Grids 2011, ser. Power Systems, A. Keyhani and M. Marwali, Eds. Springer Berlin Heidelberg, Jan. 2012, pp. 401-437. [Online]. Available: http://link.springer.com/chapter/10.1007/978-3-642-21578-0_13

[20] R. C. Dugan, "OpenDSS and ${ }^{13}$ parallel computing," Sep. $2012 . \quad$ [Online]. Available: http://sourceforge.net/p/electricdss/discussion/861976/thread/0f9191cc/\#4b55

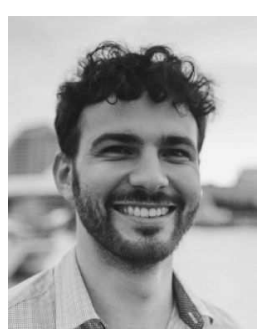

of smart grid data.

Fabian Tamp completed a B.E. (Electrical) / B. Comm. in 2013, at the University of Wollongong, Australia. He is employed as a Software Engineer at Google Australia and has previously worked as an engineer at ActewAGL, the distribution network service provider covering the Australian Capital Territory (ACT). His research interests include the transformation of electrical network data into information useful for practical decision-making, and the application of cloud computing and artificial intelligence techniques to the processing and interpretation

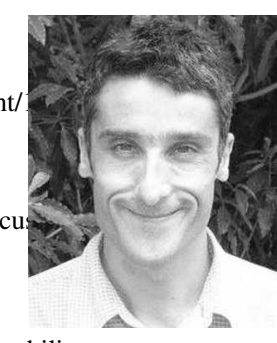

bility.
Phil Ciufo (SM'07) received the B.E. (Hons.) and M.E. (Hons.) degrees in electrical engineering from the University of Wollongong, Wollongong, Australia, in 1990 and 1993, respectively, and the Ph.D. degree in electrical engineering in 2002. Dr. Ciufo has had various stints in industry as an electrical engineer and returned to academia in 2007. His research interests include modelling and analysis of power distribution systems, advanced automation and control of distribution networks using intelligent systems and methods, and power quality and relia- 